\title{
Measurement of the Top Quark Mass using the Template Method in the Lepton plus Jets Channel With In Situ $W \rightarrow j j$ Calibration at CDF-II
}

\author{
The CDF Collaboration \\ URL http://www-cdf.fnal.gov
}

(Dated: March 4, 2006)

\begin{abstract}
We report an updated measurement of the top quark mass in the lepton plus jets channel of $t \bar{t}$ events from $p \bar{p}$ collisions at $\sqrt{s}=1.96 \mathrm{TeV}$. This measurement uses a dataset with integrated luminosity of $680 \mathrm{pb}^{-1}$, containing $360 t \bar{t}$ candidates separated into four subsamples. A top quark mass is reconstructed for each event by using energy and momentum constraints on the top quark pair decay products. We also employ the reconstructed mass of hadronic $\mathrm{W}$ boson decays $W \rightarrow j j$ to constrain in situ the largest systematic uncertainty of the top quark mass measurement: the jet energy scale. Monte Carlo templates of the reconstructed top quark and $\mathrm{W}$ boson mass are produced as a function of the true top quark mass and the jet energy scale. The distribution of reconstructed top quark and $\mathrm{W}$ boson mass in the data are compared to the Monte Carlo templates using a likelihood fit to obtain: $M_{t o p}=173.4 \pm 2.8 \mathrm{GeV} / c^{2}$.
\end{abstract}

\section{INTRODUCTION}

The direct observation of the top quark in 1995 [3] was not a big surprise since the $b$ quark was expected to have a isospin partner to insure the viability of the Standard Model. What was surprising at the time of the discovery was its large mass, almost 35 times the mass of the $b$ quark. The top quark mass is a fundamental parameter of the Standard Model, and plays an important role in the the precise prediction of electroweak observables like the Higgs boson mass. Indeed, the radiative corrections of many electroweak observables are dominated by the large top quark mass. Furthermore, a large value of the top quark mass indicates a strong Yukawa coupling to Higgs, and could be a sign for a special role of the top quark in the understanding of electroweak symmetry breaking [4]. Thus, a precise measurement of the top quark mass provides a crucial test of the consistency of the Standard Model and could help constrain physics beyond the Standard Model. In this paper, we report a measurement of the top quark mass with the CDF-II detector, using the data sample from March 2002 to August 2005 runs, corresponding to a total integrated luminosity of $680 \mathrm{pb}^{-1}$. This measurement is an update of our previous measurement $[1,2]$ which used the same method with $318 \mathrm{pb}^{-1}$ of data.

At the Tevatron, top quarks are produced primarily as $t \bar{t}$ pairs and decay to $W$ bosons and $b$ quarks nearly $100 \%$ of the time within the Standard Model. Then, the $W$ bosons can decay into lepton-neutrino $(l \nu)$ or quark pairs $\left(q q^{\prime}\right)$. In this measurement, we use the "lepton+jet" channel of $t \bar{t}$ candidates in which only one of two W bosons decays to $l \nu$ while the other decays to quark pairs.

The template method relies on good Monte Carlo modeling of $t \bar{t}$ and background events. We generate a set of Monte Carlo samples at a range of true top quark masses $\left(\mathrm{M}_{\text {top }}\right)$ and a range of jet energy scale calibrations shifts $\left(\Delta_{\text {JES }}\right)$. More details on the jet energy scale calibrations and the $\Delta_{\text {JES }}$ parameter are given in Sec. III. We form good estimators of both of these parameters: reconstructed top mass $m_{t}^{\text {reco }}$ and $q q^{\prime}$ dijet mass $m_{\mathrm{jj}}$. The reconstructed top mass $m_{t}^{\text {reco }}$ is extracted from each event using a $\chi^{2}$ fitter. The $\chi^{2}$ fitter considers all possible jet-to-parton assignment hypotheses and places constraints on the quantities in the event. Most notably it incorporates $W$ mass constraint and a requirement that $t$ quarks on both leptonic and hadronic sides are reconstructed with the same mass $m_{t}^{\text {reco. }}$. We compute the $m_{t}^{\text {reco }}$ and $m_{\mathrm{jj}}$ distributions or "templates" from all the $\mathrm{M}_{\mathrm{top}}$ and $\Delta_{\mathrm{JES}}$ samples. Measurement of $\mathrm{M}_{\mathrm{top}}$ and the in situ jet energy scale calibration $\Delta_{\mathrm{JES}}$ is performed by comparing the $m_{t}^{\text {reco }}$ and $m_{\mathrm{jj}}$ distributions obtained from the data to these templates using an unbinned likelihood fit. 


\section{EVENT SELECTION}

The lepton+jets events are selected by requiring one well-identified electron or muon, large $\mathbb{E}_{T}$ due to the neutrino from the $\mathrm{W}$ decay and at least four jets in the final state.

Electron candidates are identified as a high-momentum track in the tracking system matched to an electromagnetic cluster reconstructed in the calorimeters with $E_{T}>20 \mathrm{GeV}$. The ratio of hadronic to electromagnetic energy deposition in the cluster is required to be low to ensure validity of the electron hypothesis. We also require that energy shared by the towers surrounding the cluster is low. Muon candidates are reconstructed as high-momentum tracks with $p_{T}>20$ $\mathrm{GeV} / \mathrm{c}$ matching hits in the muon chambers. Energy deposited in the calorimeter is required to be consistent with a minimum ionizing particle.

The missing transverse energy is measured by the imbalance in the calorimeter transverse energy and is required to be greater than $20 \mathrm{GeV}$.

Jets are reconstructed with the JETCLU cone algorithm with a radius $R=\sqrt{\eta^{2}+\phi^{2}}=0.4$. At least 4 jets are required with the jet $E_{T}$ requirement depending on the event category as described below.

A final requirement is applied only for the top quark mass reconstruction: the minimized $\chi^{2}$ value from the kinematic fit described in Sec. IV is required to be less than 9. We use the reconstructed W boson mass also from events that failed that cut since $\mathrm{W}$ mass constraint is used in the fit.

To improve the statistical power of the method, the lepton+jets sample is divided into four subsamples with various sensitivity to the top quark mass. First the events are separated based on the number of jets that are b-tagged in the event. The SECVTX algorithm [6] based on the identification of secondary vertices inside jets is used to tag b-jets. Events with 2, 1 and no tags are considered separately. Events with increasing number of b-tags have better mass resolution (as described in Sec. IV) and lower background contamination. Furthermore, events with 1-tag are separated based on the 4 th jet $E_{T}$ threshold. Events in the 1-tag(T) category have 4 jets with $E_{T}>15 \mathrm{GeV}$, while events in the $1-\operatorname{tag}(\mathrm{L})$ category have 3 jets with $E_{T}>15 \mathrm{GeV}$ and the 4 th jet with $15>E_{T}>8 \mathrm{GeV}$. Events in the 1-tag $(\mathrm{T})$ sample are less contaminated by background. Table I describes the four subsamples with their expected signal to background ratio and the number of events observed in data (before and after the $\chi^{2}$ cut).

\begin{tabular}{ccccc}
\hline \hline Category & 2-tag & 1-tag $(\mathrm{T})$ & 1-tag (L) & 0-tag \\
\hline \hline leading 3 jets $E_{T}$ & $>15 \mathrm{GeV}$ & $>15 \mathrm{GeV}$ & $>15 \mathrm{GeV}$ & $>21 \mathrm{GeV}$ \\
\hline 4th jet $E_{T}$ & $>8 \mathrm{GeV}$ & $>15 \mathrm{GeV}$ & $>8 \mathrm{GeV}$ & $>21 \mathrm{GeV}$ \\
& & & $<15 \mathrm{GeV}$ & \\
\hline Expected S:B & $10.6: 1$ & $3.7: 1$ & $1.1: 1$ & no a-priori estimate \\
\hline Number of events & 57 & 120 & 75 & 108 \\
\hline events with $\chi^{2}<9$ & 38 & 105 & 61 & 97
\end{tabular}

TABLE I: Jet $E_{T}$ cut and b-tagging requirement for the 4 event categories. Also shown is the expected signal to background ratio (S:B) for each subsample as well as the number of events observed in data before and after the $\chi^{2}$ cut. Note that we do not use any a priori background estimates for the 0-tag subsample.

\section{JET ENERGY SCALE}

We describe in this section the a priori determination of the jet energy scale uncertainty by CDF that is used later in this analysis. There are many sources of uncertainties related to jet energy scale at CDF:

- Relative response of the calorimeters as a function of pseudorapidity with respect to the central calorimeter.

- Single particle response in the calorimeters.

- Fragmentation of jets.

- Modeling of the underlying event energy.

- Amount of energy deposited out-of-cone.

The uncertainty on each source is evaluated separately as a function of the jet $p_{T}$ (and $\eta$ for the first uncertainty in the list above). Their contributions are shown in Fig. 1 for the region $0.2<\eta<06$. The black lines show the sum in quadrature $\left(\sigma_{c}\right)$ of all contributions. This $\pm 1 \sigma_{c}$ total uncertainty is taken as a unit of of jet energy scale miscalibration $\left(\Delta_{J E S}\right)$ in this analysis. 


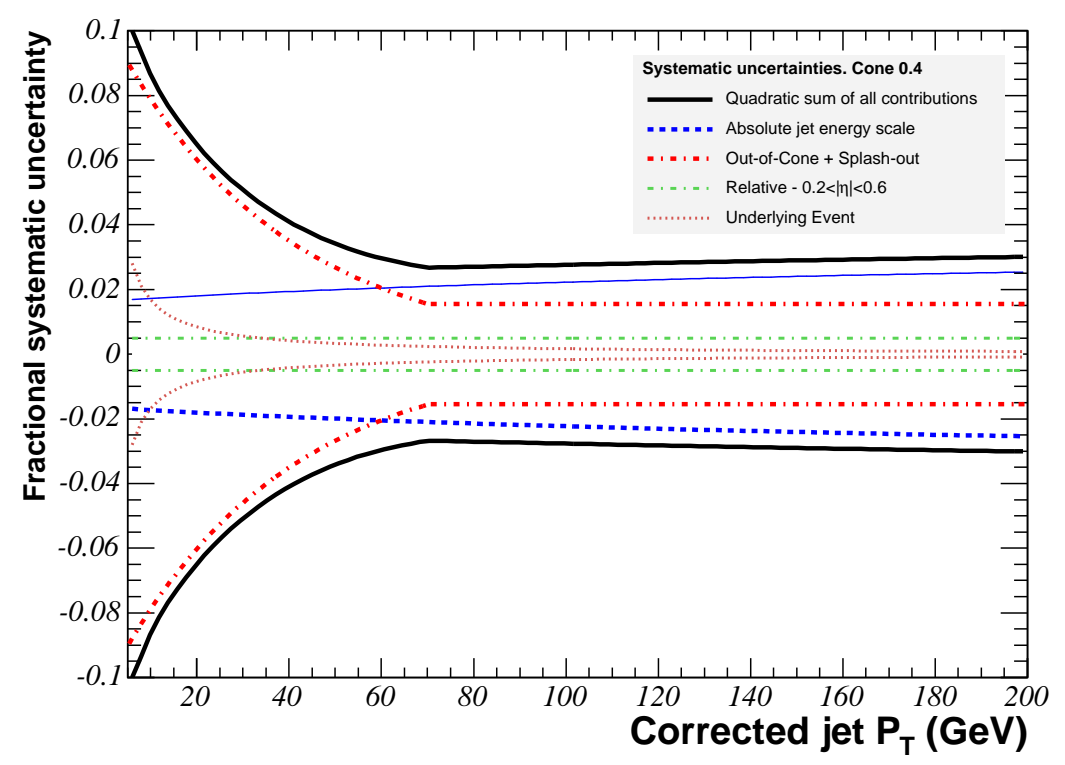

FIG. 1: Jet energy scale uncertainty as a function of the corrected jet $p_{T}$ for the underlying event (dotted red), relative response (dashed green), out-of-cone energy (dashed red) and absolute response (dashed blue). The contribution of all sources are added in quadrature (full black) to form the total $\Delta_{J E S}$ systematic $\sigma_{c}$

\section{TOP QUARK MASS RECONSTRUCTION}

For each lepton+jets event, an invariant mass of the top quark is reconstructed from the top decay products (lepton candidate, four highest $E_{T}$ jets and missing transverse energy) using a $\chi^{2}$ kinematic fit. Reconstructed top quark mass distributions are produced using HERWIG [5] Monte Carlo events for various true top quark mass and $\Delta_{\text {JES }}$ hypothesis. These distributions are called top quark mass templates and have a strong dependence on the true top quark mass. As discussed in more details in the following section, we also construct $\mathrm{W}$ boson dijet mass templates.

The purpose of the $\chi^{2}$ kinematic fit is to extract a single good estimator of the true top quark mass from all information available in the event. Inputs to the fitter include lepton and jet four-vectors (together with the b-tagging information) as well as the unclustered energy. The $\chi^{2}$ as presented in Eqn. IV.1 is minimized for all jet-to-parton assignments consistent with the b-tagging information. For each such combination two neutrino $p_{z}$ solutions consistent with the $W$ mass on the leptonic side exist. The fit is performed with both $p_{z}$ solutions taken as an initial condition (neutrino $p_{z}$ is a free parameter in the fit). Precise definition of $\chi^{2}$ is:

$$
\begin{aligned}
\chi^{2}= & \sum_{i=\ell, 4 j e t s} \frac{\left(p_{T}^{i, f i t}-p_{T}^{i, \text { meas }}\right)^{2}}{\sigma_{i}^{2}} \\
& +\sum_{j=x, y} \frac{\left(p_{j}^{U E, f i t}-p_{j}^{U E, \text { meas }}\right)^{2}}{\sigma_{j}^{2}} \\
& +\frac{\left(M_{\ell \nu}-M_{W}\right)^{2}}{\Gamma_{W}^{2}}+\frac{\left(M_{j j}-M_{W}\right)^{2}}{\Gamma_{W}^{2}} \\
& +\frac{\left(M_{b \ell \nu}-m_{t}^{\mathrm{reco}}\right)^{2}}{\Gamma_{t}^{2}}+\frac{\left(M_{b j j}-m_{t}^{\mathrm{reco}}\right)^{2}}{\Gamma_{t}^{2}},
\end{aligned}
$$

where $\sigma_{l}, \sigma_{j}$ and $\sigma_{x, y}$ are the uncertainties on the lepton, jets and the unclustered energy respectively. The first two terms constrain the fitted lepton and jet momenta and components of unclustered energy to their measured values. The third and fourth terms provide the most powerful constraint in the fit: the invariant mass of neutrino and lepton and the invariant mass of light quark jets are constrained to the measured mass of $\mathrm{W}$ boson. The last two terms enforce the requirement that the reconstructed top mass $m_{t}^{\text {reco }}$ is the same on the leptonic and hadronic legs of the decay. Note that for each jet-to-parton assignment hypothesis flavour-dependent jet energy corrections are applied. 


\section{CDF Run II Preliminary}
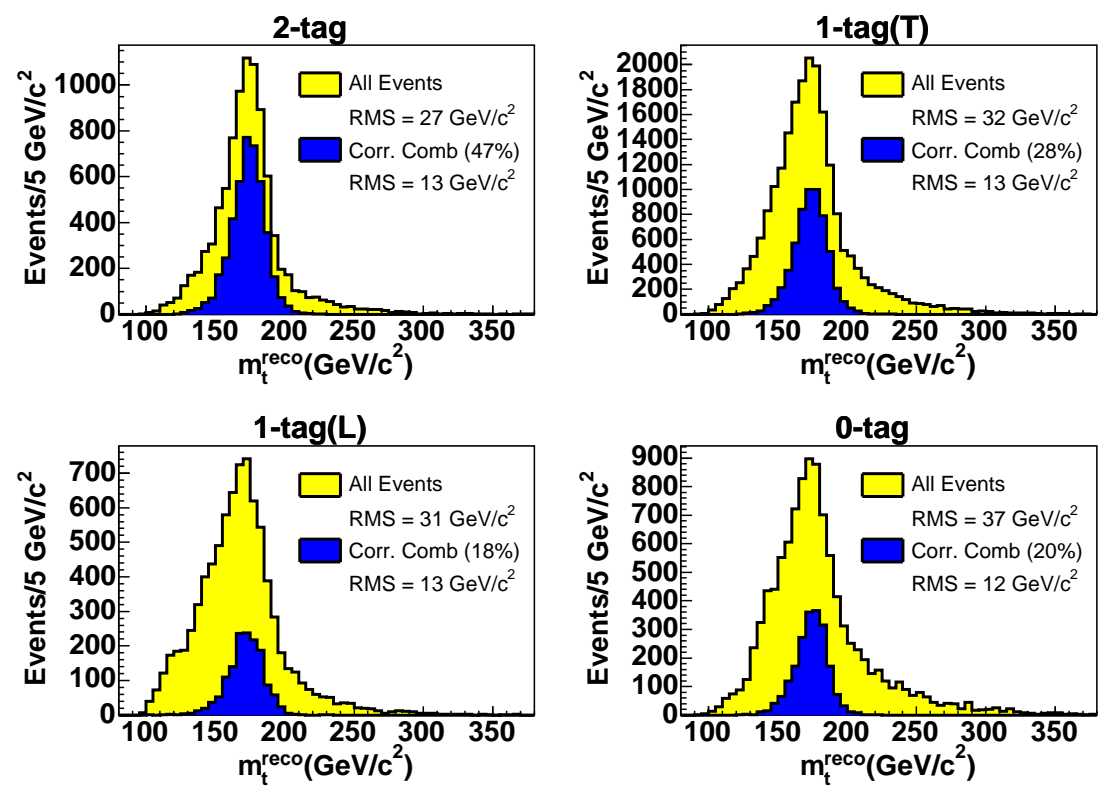

FIG. 2: Reconstructed top quark mass for 2-tag (upper left), 1-tag(T) (upper right), 1-tag(L) (bottom left) and 0-tag (bottom right) signal events $\left(M_{t o p}=178 \mathrm{GeV} / \mathrm{c} 2, \Delta_{J E S}=0\right)$. Dark blue histogram shows the $m_{t}^{\text {reco }}$ distribution in the events where the parton-to-jet assigment chosen by the fitter is consistent with Monte Carlo truth.

We use $m_{t}^{\text {reco }}$ from the fit that yields the lowest $\chi^{2}$, provided that $\chi^{2}<9$. Figure 2 shows the reconstructed top mass distributions with overlayed $m_{t}^{\text {reco }}$ distribution for the events where the parton-to-jet assigment chosen by the fitter is consistent with Monte Carlo truth.

\section{W BOSON MASS RECONSTRUCTION}

To perform in situ jet energy scale calibration we construct the dijet mass $m_{\mathrm{jj}}$. This is simply the invariant mass of the measured jets pairs which are not b-tagged. Note that in an event where one or fewer $b$ tags are present it is possible to form more than one $m_{\mathrm{jj}}$. In such cases all values are used. Figure 3 shows the $m_{\mathrm{jj}}$ distributions for all subsamples, where the blue subset shows the jet pairs that are matched to the $W$ daughter partons.

\section{SIGNAL TEMPLATE PARAMETRIZATION}

Monte Carlo samples are available only at discrete values of true $\mathrm{M}_{\text {top }}$ and $\Delta_{\mathrm{JES}}$. We parametrize the $m_{t}^{\text {reco }}$ and $m_{\mathrm{jj}}$ distributions as a function of two parameters $\mathrm{M}_{\mathrm{top}}$ and $\Delta_{\mathrm{JES}}$ to obtain two probability density functions $P_{s}\left(m_{t}^{\text {reco }} ; \mathrm{M}_{\mathrm{top}}, \Delta_{\mathrm{JES}}\right)$ and $P_{s}\left(m_{\mathrm{jj}} ; \mathrm{M}_{\mathrm{top}}, \Delta_{\mathrm{JES}}\right)$ that depend on two parameters $\mathrm{M}_{\mathrm{top}}$ and $\Delta_{\mathrm{JES}}$. An example of the dependence of $P_{s}\left(m_{t}^{\text {reco }} ; \mathrm{M}_{\mathrm{top}}, \Delta_{\mathrm{JES}}\right)$ on the true top mass and dependence of $P_{s}\left(m_{\mathrm{jj}} ; \mathrm{M}_{\mathrm{top}}, \Delta_{\mathrm{JES}}\right)$ on the assumed $\Delta_{\mathrm{JES}}$ is shown in Fig. 4.

\section{BACKGROUND TEMPLATES}

An a priori estimate for background composition (Table II) is used to obtain $m_{t}^{\text {reco }}$ and $m_{\mathrm{jj}}$ shapes for background. We use $W b b+2$ partons HERWIG Monte Carlo samples to model all $W+$ heavy flavour as well as the diboson backgrounds. The QCD bacground is modeled with ALPGEN [7] $W+$ light flavour samples. Careful studies revealed that these samples model backgrounds well. Note that the background shapes are assumed to be independent of the $\mathrm{M}_{\text {top }}$ and $\Delta_{\mathrm{JES}}$ parameters. Slight dependence on $\Delta_{\mathrm{JES}}$ is taken as a source of systematic uncertainty. The composite 


\section{CDF Run II Preliminary}
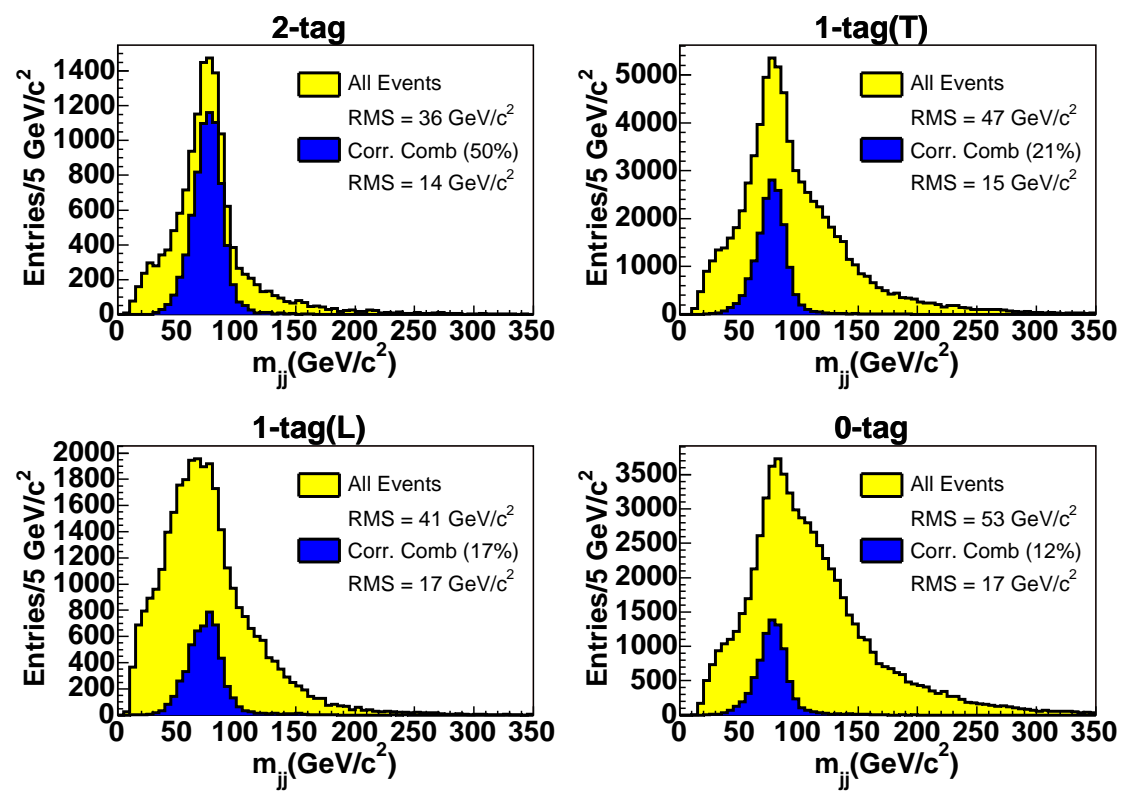

FIG. 3: $m_{\mathrm{jj}}$ distributions for 2-tag (upper left), 1-tag(T) (upper right), 1-tag(L) (bottom left) and 0-tag (bottom right) signal events $\left(M_{t o p}=178 \mathrm{GeV} / \mathrm{c} 2, \Delta_{J E S}=0\right)$. The blue subset shows the jet pairs that are matched to the $W$ daughter partons.

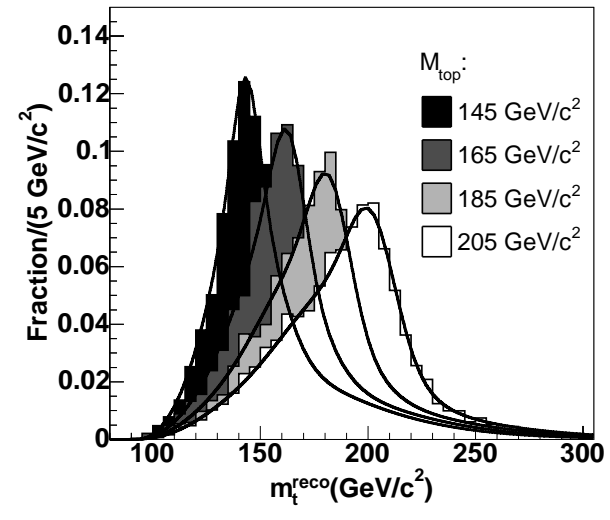

a)

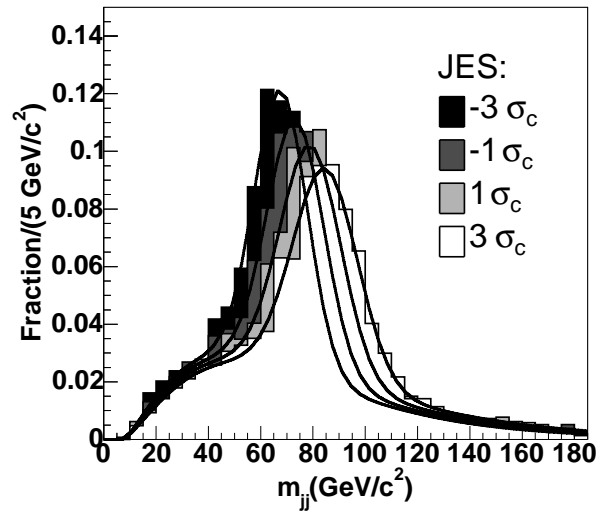

b)

FIG. 4: a) 1-tag(T) $m_{t}^{\text {reco }}$ templates and parametrizations for a range of true top masses at $\Delta_{\mathrm{JES}}=0$ b) 2 -tag $m_{\mathrm{jj}}$ templates for several values of $\Delta_{\mathrm{JES}}$ and $\mathrm{M}_{\mathrm{top}}$ fixed at $180 \mathrm{GeV} / \mathrm{c}^{2}$

background $m_{t}^{\text {reco }}$ and $m_{\mathrm{jj}}$ distributions are parametrized to obtain the probability distribution functions $P_{B}\left(\mathrm{M}_{\mathrm{top}}\right)$ and $P_{B}\left(m_{\mathrm{jj}}\right)$. Background templates and parametrization shapes are shown in Figure 5.

\section{LIKELIHOOD FIT}

The reconstructed mass distributions from data are compared to the signal and background templates using an unbinned likelihood fit. The likelihood fits for the expectation values of the number of signal and background events in each subsample, and for the top quark pole mass and jet energy scale. For each subsample, the likelihood is given 


\begin{tabular}{ccccc}
\hline \hline & 2-tag & 1-tag(T) & 1-tag(L) & 0-tag \\
\hline \hline Non-w(QCD) & $0.6 \pm 0.2$ & $5.0 \pm 1.4$ & $4.4 \pm 1.4$ & \\
\hline W+Heavy Flavor & $2.4 \pm 1.0$ & $8.4 \pm 3.0$ & $14.6 \pm 4.7$ & \\
\hline W+light jets & $0.9 \pm 0.2$ & $6.9 \pm 1.4$ & $8.9 \pm 1.9$ & $100 \%$ \\
\hline WW/WZ & $0.11 \pm 0.03$ & $1.0 \pm 0.3$ & $1.5 \pm 0.4$ & \\
\hline Single Top & $0.02 \pm 0.01$ & $1.1 \pm 0.3$ & $1.3 \pm 0.3$ & \\
\hline \hline Total Background & $4.0 \pm 1.3$ & $22.2 \pm 4.7$ & $30.6 \pm 6.7$ & \\
\hline \hline$t \bar{t}\left(\sigma_{t \bar{t}}=6.1 \mathrm{pb}\right)$ & 42.8 & 82.2 & 33.6 & \\
\hline \hline Total & 46.8 & 104.4 & 64.2 & \\
\hline \hline
\end{tabular}

TABLE II: Background composition and signal estimate for all subsamples. Note that in the 0-tag subsample the background is dominated by $W+$ light jets, but a priori overall normalization is not used

by:

$$
\mathcal{L}_{\text {sample }}=\mathcal{L}_{\text {shape }}^{m_{t}^{\text {reco }}} \times \mathcal{L}_{\text {shape }}^{m_{\text {jj }}} \times \mathcal{L}_{\text {nev }} \times \mathcal{L}_{\text {bg }}
$$

where

$$
\begin{aligned}
& \mathcal{L}_{\text {shape }}^{m_{t}^{\text {reco }}}=\prod_{k=1}^{N^{\chi^{2}}} \frac{\left[\epsilon_{s} n_{s} P_{s}\left(m_{t, k}^{\mathrm{reco}} ; \mathrm{M}_{\mathrm{top}}, \Delta_{\mathrm{JES}}\right)+\epsilon_{b} n_{b} P_{b}\left(m_{t, k}^{\mathrm{reco}}\right)\right]}{\epsilon_{s} n_{s}+\epsilon_{b} n_{b}} ; \\
& \mathcal{L}_{\text {shape }}^{m_{\mathrm{jj}}}=\prod_{k=1}^{N \cdot C_{i}} \frac{n_{s} P_{s}\left(m_{\mathrm{jj}, \mathrm{k}} ; \mathrm{M}_{\mathrm{top}}, \Delta_{\mathrm{JES}}\right)+n_{b} P_{b}\left(m_{\mathrm{jj}, \mathrm{k}}\right)}{n_{s}+n_{b}} ; \\
& \mathcal{L}_{\text {nev }}=\sum_{N_{s}+N_{b}=N} \operatorname{Pois}\left(N_{s} ; n_{s}\right) \times \operatorname{Pois}\left(N_{b} ; n_{b}\right) \times \\
& {\left[\sum_{N_{s}^{\chi^{2}}+N_{b}^{\chi^{2}}=N \chi^{2}}^{N_{s, b}^{\chi^{2}} \leq N_{s, b}} B\left(N_{s}^{\chi^{2}} ; N_{s}, \epsilon_{s}\right) B\left(N_{b}^{\chi^{2}} ; N_{b}, \epsilon_{b}\right)\right]} \\
& \mathcal{L}_{\mathrm{bg}}=\exp \left(-\frac{\left(n_{b}-n_{b}^{0}\right)^{2}}{2 \sigma_{n_{b}}^{2}}\right) \text {. }
\end{aligned}
$$

The free parameters in the fit are $\mathrm{M}_{\mathrm{top}}, \Delta_{\mathrm{JES}}, n_{b}$ and $n_{s}$-top mass, jet energy scale shift, expected number of background events and expected number of signal events.

The values $\epsilon_{s}$ and $\epsilon_{b}$ represent the efficiency of the $\chi^{2}$ cut for signal and background events, respectively. $N$ and $N^{\chi^{2}}$ are the number of events observed in the data before and after the $\chi^{2}$ cut.

The $k$ th term of $\mathcal{L}_{\text {shape }}^{m_{t}^{\text {reco }}}$ gives the probability of observing the $k$ th data event with reconstructed mass $m_{t, k}^{\text {reco }}$ given $\mathrm{M}_{\text {top }}$ and $\Delta_{\text {JES }}$ values. This term is the most sensitive to fitted $\mathrm{M}_{\text {top }}$ value.

Similarly $\mathcal{L}_{\text {shape }}^{m_{\mathrm{jj}}}$ is sensitive to $\Delta_{\mathrm{JES}}$ value. Note that all the $m_{\mathrm{jj}}$ contributions from different dijet combinations $C_{i}$ are taken in the product.

The third term in the likelihood, $\mathcal{L}_{\text {nev }}$, captures the information arising from the number of signal and background events in the top quark mass and dijet mass samples, which are correlated. The number of observed events $N$ and number of events passing $\chi^{2}$ cut $N^{\chi^{2}}$ are known, but we don't know how many signal $N_{s}$ and background $N_{b}$ events exist within the sample. Also unknown are $N_{s}^{\chi^{2}}$ and $N_{b}^{\chi^{2}}$ - numbers of signal and background passing $\chi^{2}$ cut. We sum over all possibilities of assigning $N_{s}, N_{b}, N_{s}^{\chi^{2}}$ and $N_{b}^{\chi^{2}}$ and calculate the probability of such assignment given $n_{s}$ and $n_{b}$

The $\mathcal{L}_{\text {bg }}$ term is a Gussian constraint on the expected number of background events.

The total likelihood is a product of likelihoods for all subsamples with a Gaussian constraint on $\Delta_{\text {JES }}$ with mean of 0 and width of 1 . This constraint is imposed since we trust our a priori jet energy scale calibration to within $\pm 1 \sigma$. 

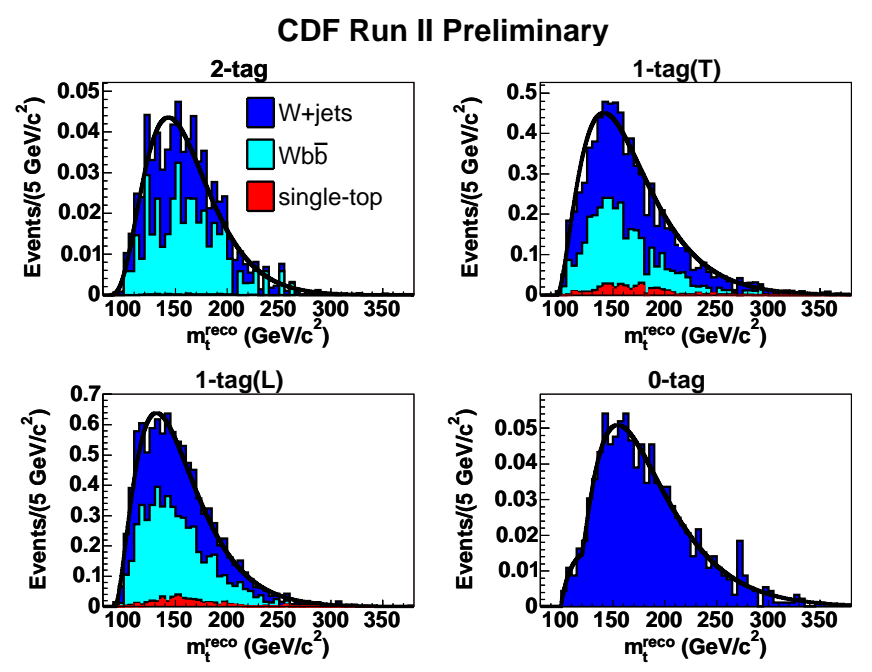

a)

CDF Run II Preliminary
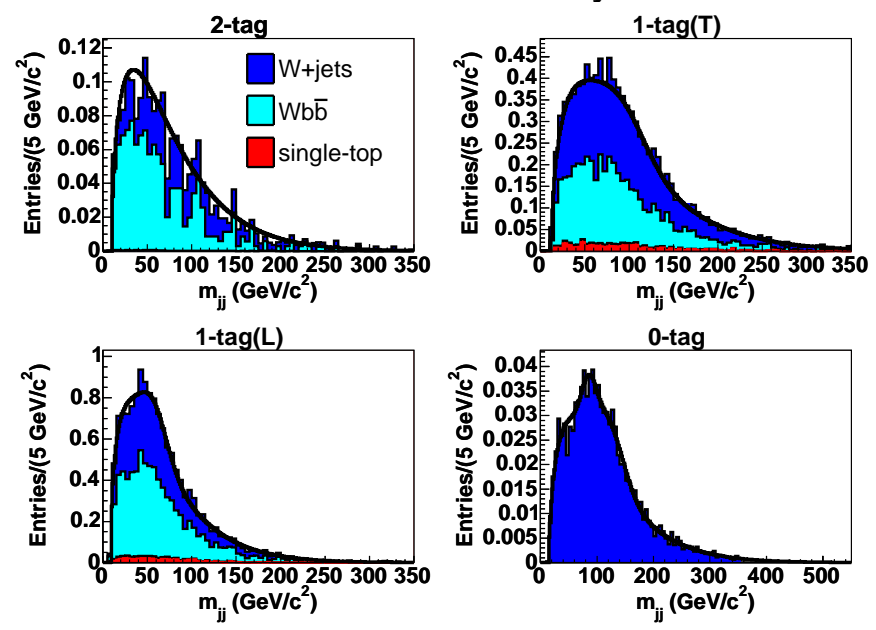

b)

FIG. 5: a) $m_{t}^{\text {reco }}$ background template and parametrization shape b) $m_{\mathrm{jj}}$ background template and parametrization shape

\section{METHOD CHECK}

We test the procedure by performing 3000 pseudoexperiments at a mesh of points with $\Delta_{\text {JES }}$ between -2.0 and 2.0 $\sigma_{c}$ and $\mathrm{M}_{\text {top }}$ between 165 and $180 \mathrm{GeV} / \mathrm{c}^{2}$. Figures $6 \mathrm{a}$ ), $6 \mathrm{~b}$ ), 7 a) and $7 \mathrm{~b}$ ) show respectively the $\mathrm{M}_{\text {top }}$ residuals, $\Delta_{\text {JES }}$ residuals, $\mathrm{M}_{\text {top }}$ pull widths and $\Delta_{\text {JES }}$ pull widths. The Monte Carlo samples we use to estimate the bias of the method are statistically limited at the $\sim 0.3 \mathrm{GeV}$ level, making it difficult to resolve smaller effects; averaging over the points in Fig. 6a), we conclude the method is unbiased. The pull widths are slightly larger than one, due to the non-Gaussian nature of our likelihood. We scale the reported statistical uncertainty by a factor of 1.019 to account for this effect in the final result.

\section{RESULTS}

The likelihood procedure when applied to data yields:

$$
\begin{gathered}
\mathrm{M}_{\mathrm{top}}=173.4 \pm 2.5\left(\text { stat }+\Delta_{\mathrm{JES}}\right) \mathrm{GeV} / c^{2} \\
\Delta_{\mathrm{JES}}=-0.31_{-0.58}^{+0.59}\left(\mathrm{stat}+\mathrm{M}_{\mathrm{top}}\right) \sigma_{c}
\end{gathered}
$$




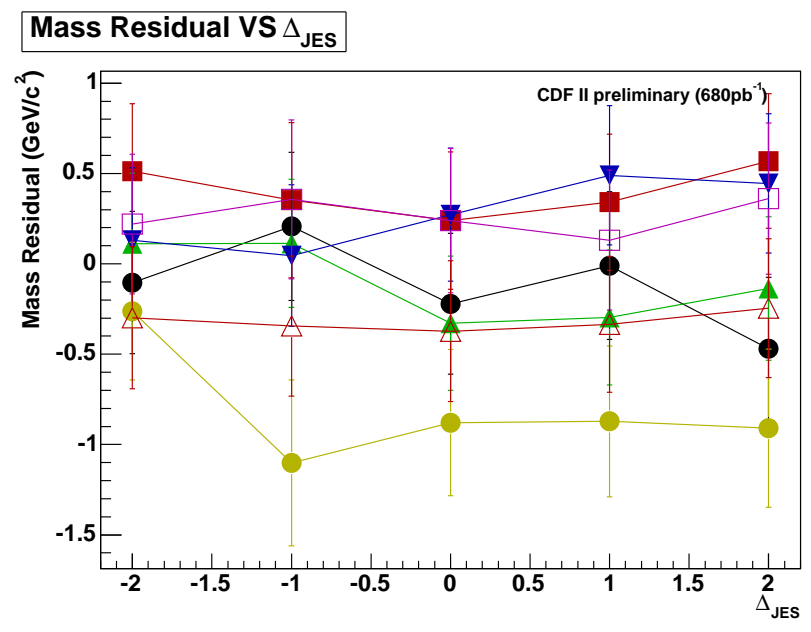

a)

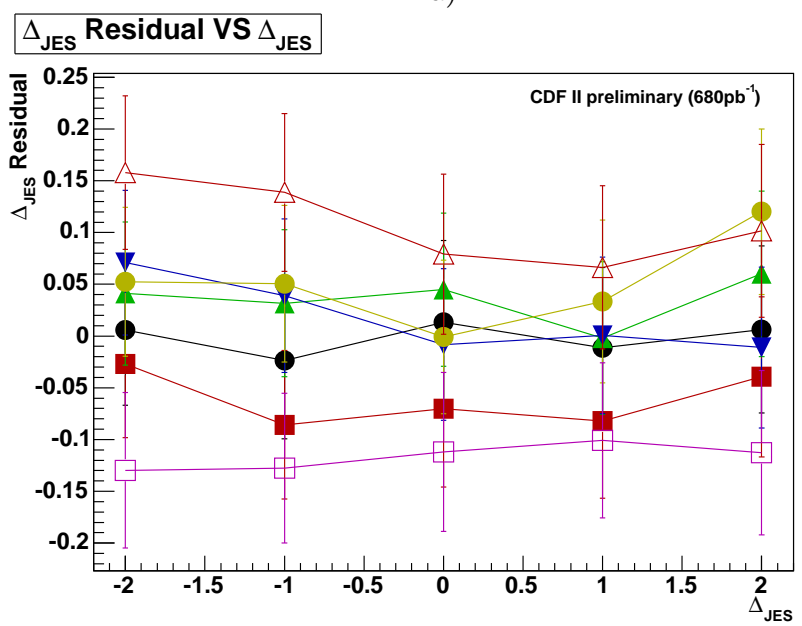

b)

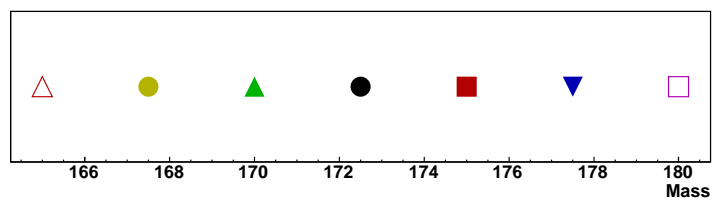

FIG. 6: $\mathrm{M}_{\text {top }}$ (a) and $\Delta_{\mathrm{JES}}$ (b) residuals for a set of mass points VS $\Delta_{\text {JES }}$.

We can approximately disentangle the $\Delta_{\text {JES }}$ contribution to the uncertainy on $\mathrm{M}_{\text {top }}$ by performing the likelihood fit with $\Delta_{\text {JES }}$ fixed to the global minimum value, obtaining pure statistical uncertainty. The remaining part of the $2.5 \mathrm{GeV}$ uncertainty is due to allowed variations in $\Delta_{\text {JES }}$. The result is:

$$
\mathrm{M}_{\text {top }}=173.4 \pm 1.7 \text { (stat) } \pm 1.8\left(\Delta_{\mathrm{JES}}\right) \mathrm{GeV} / c^{2}
$$

Fit constraints and results are summarized in Table III. Negative log likelihood contours are depicted in Fig. 8. Each oval corresponds approximately to a single standard deviation distance from the $\mathrm{M}_{\text {top }}, \Delta_{\text {JES }}$ values preferred by the fit. Figures 9 and 10 show the $m_{t}^{\text {reco }}$ and $m_{\mathrm{jj}}$ distributions in data and the signal and background probability distribution functions evaluated at the fitted $\mathrm{M}_{\text {top }}$ and $\Delta_{\text {JES }}$ and normalized to the fitted $n_{s}$ and $n_{b}$ values. 


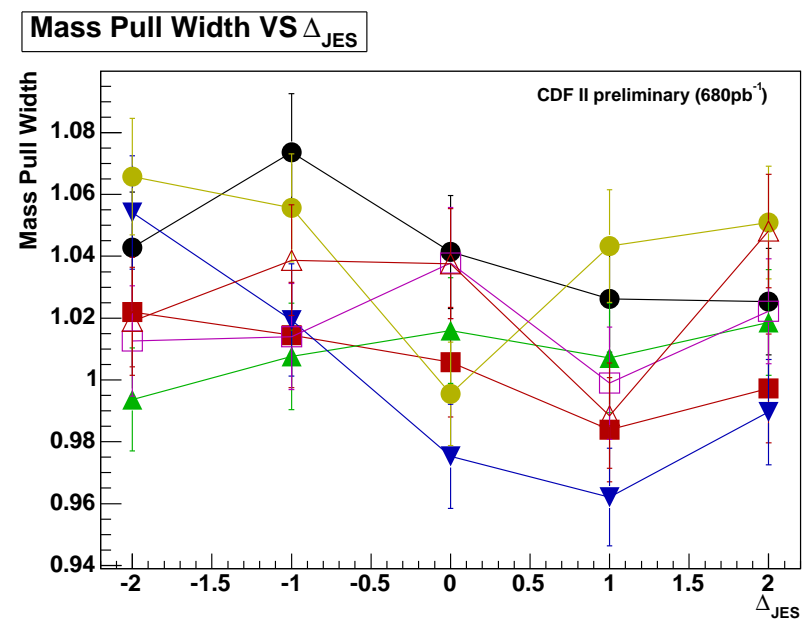

a)

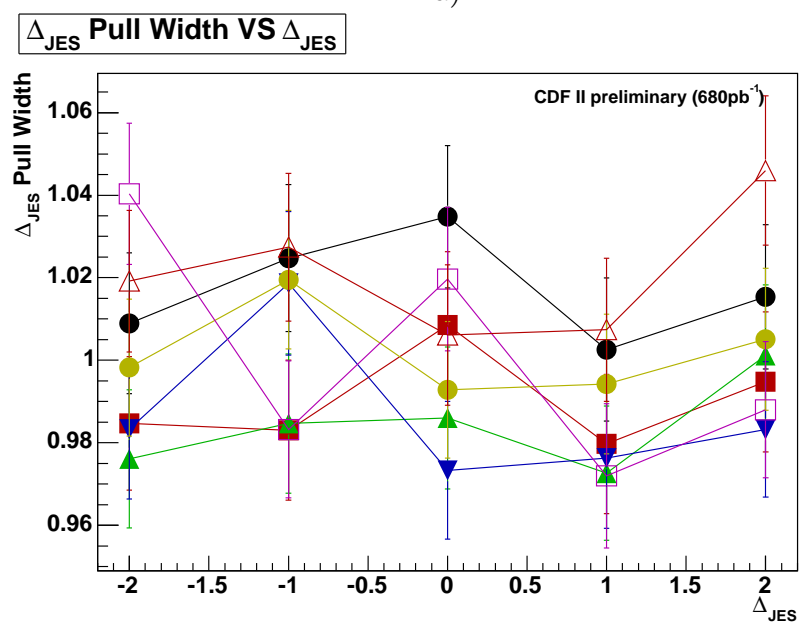

b)

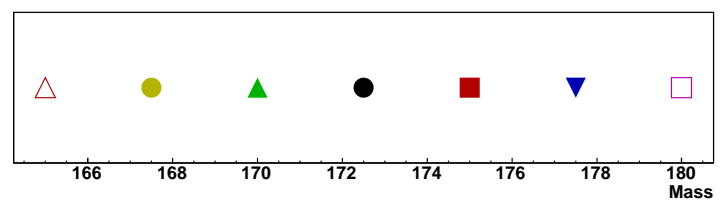

FIG. 7: $\mathrm{M}_{\text {top }}(\mathrm{a})$ and $\Delta_{\mathrm{JES}}(\mathrm{b})$ pull widths VS $\Delta_{\mathrm{JES}}$.

To ascertain how likely it is that we obtain the statistical error that we observe in the data we perform a set of pseudoexperiments with total number of events in a pseudoexperiment matching number of events observed in data. As illustrated in Figure 11, we estimate that smaller than observed error can occur approximately $4 \%$ of the time.

\section{SYSTEMATIC UNCERTAINTIES}

Here we provide only a brief summary of the sources of systematic uncertainty ( $c f$ table IV). Please refer to [1] for an in-depth discussion of each of the uncertainties.

The $b$ jets can behave differently than gluon and light quark jets because of their different fragmentation models, more abundant semi leptonic decays and different color flow in $t \bar{t}$ events. We find that the uncertainties due to the unique features of the $b$ jet are $0.6 \mathrm{GeV} / c^{2}$.

The parameter $\Delta_{\text {JES }}$ does not fully capture complexities of jet energy scale uncertainties. In particular, component 


\begin{tabular}{|c|c|c|c|c|c|}
\hline \multicolumn{2}{|c|}{ Category } & 2 -tag & 1-tag $(\mathrm{T})$ & $1-\operatorname{tag}(\mathrm{L})$ & 0 -tag \\
\hline $\mathrm{M}_{\text {top }}$ & $\begin{array}{l}\text { constr. } \\
\text { fit }\end{array}$ & \multicolumn{4}{|c|}{$\begin{array}{c}\mathbf{1 7 3 . 4} \pm \mathbf{2 . 5}\left(\text { stat. }+\boldsymbol{\Delta}_{\mathrm{JES}}\right) \mathbf{G e V} / \mathbf{c}^{\mathbf{2}} \\
\left(173.4 \pm 1.7(\text { stat. }) \pm 1.8\left(\Delta_{\mathrm{JES}}\right) \mathrm{GeV} / c^{2}\right)\end{array}$} \\
\hline JES & $\begin{array}{l}\text { constr. } \\
\text { fit } \\
\text { constr. }\end{array}$ & \multicolumn{4}{|c|}{$\begin{array}{c}0.0 \pm 1.0 \sigma_{c} \\
-0.31_{-0.58}^{+0.59} \sigma_{c} \\
\text { None }\end{array}$} \\
\hline & fit & $54.0 \pm 7.1$ & $101.0 \pm 11.2$ & $41.7 \pm 8.6$ & $67.4 \pm 12.5$ \\
\hline$n_{b}$ & $\begin{array}{l}\text { constr. } \\
\text { fit }\end{array}$ & $\begin{aligned} 4.04 & \pm 1.26 \\
3.7 & \pm 1.2\end{aligned}$ & $\begin{array}{l}22.22 \pm 4.68 \\
20.3_{-4.3}^{+4.4}\end{array}$ & $\begin{array}{c}30.58 \pm 6.73 \\
32.2_{-5.4}^{+5.5}\end{array}$ & $3 \begin{array}{c}\text { None } \\
40.6_{-11.1}^{+12.0}\end{array}$ \\
\hline
\end{tabular}

TABLE III: Summary of the constraints and fit results for $\mathrm{M}_{\mathrm{top}}, \Delta_{\mathrm{JES}}, n_{b}$ and $n_{s}$

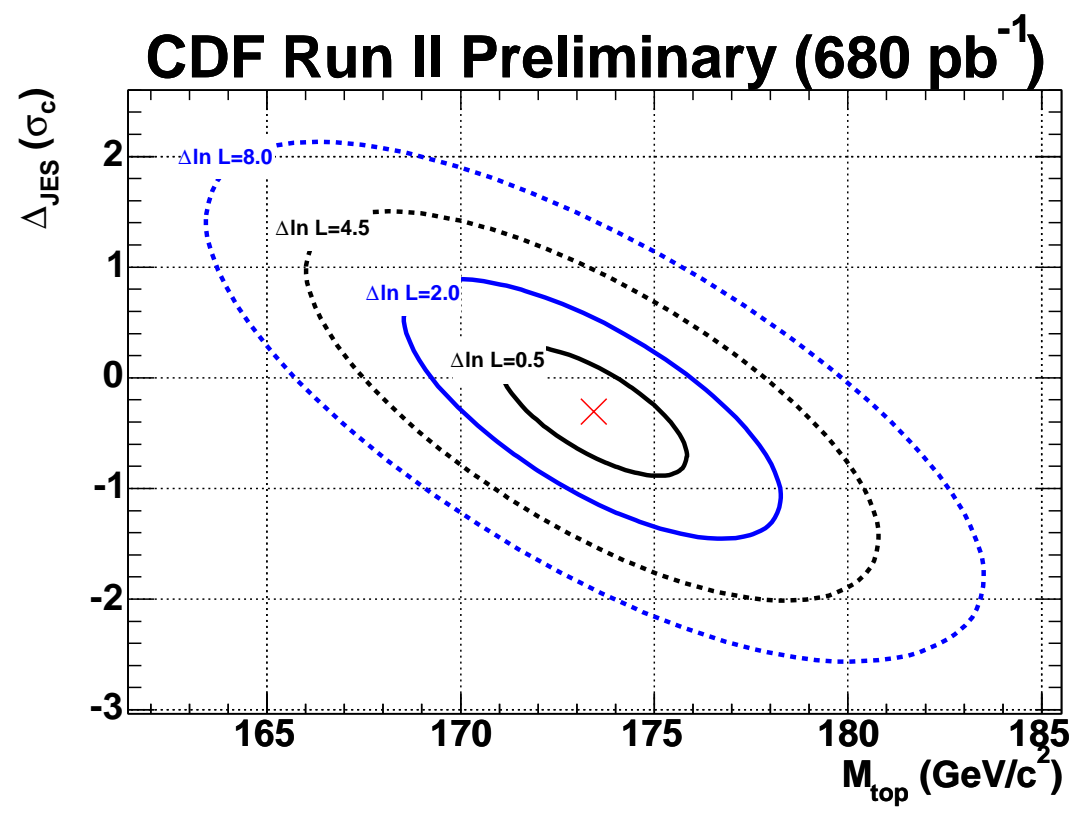

FIG. 8: Likelihood contours at successive $1 \sigma$ distances (in the Gaussian approximation) from the fitted value.

uncertainties have different $p_{T}$ and $\eta$ dependencies. Fitting for $\Delta_{\text {JES }}$ will not entirely eliminate the jet energy scale uncertainties giving rise to the residual JES uncertainty. To evaluate this uncertainty we apply variations of jet energy calibrations due to the known effects separately and add the resulting shifts in $\mathrm{M}_{\text {top }}$ in quadrature obtaining $0.7 \mathrm{GeV} / c^{2}$ for the Residual JES component.

The initial and final state gluon radiation is estimated by studying the transverse momentum of Drell-Yan events and extrapolating the results to the $Q^{2}$ of a $t \bar{t}$ event. Uncertainties of 0.5 and $0.2 \mathrm{GeV} / c^{2}$ on $\mathrm{M}_{\text {top }}$ are estimated for the initial and final state radiation, respectively.

The uncertainties in the parton distribution functions (PDF) are estimated by using different PDF sets (CTEQ5L vs MRST72), different values of $\Lambda_{\mathrm{QCD}}$ and varying the eigenvectors of the CTEQ6M set, yielding a total uncertainty of $0.3 \mathrm{GeV} / c^{2}$. The difference in fitted Mtop for mass distributions constructed using the Pythia [8] and HERWIG generators is evaluated to be $0.2 \mathrm{GeV} / c^{2}$ and is taken as a generator uncertainty. Background JES systematic uncertainty (evaluated at $0.4 \mathrm{GeV} / c^{2}$ ) result from the assumption that the background shapes are modeled as independent of $\Delta_{\text {JES }}$.

The uncertainty in the background mass shape is dominated by the $Q^{2}$ scale used in the generation of $\mathrm{W}+$ jets events. ALPGEN samples with various $Q^{2}$ scales are used to extract different background mass templates. The Shifts in the templates introduce an uncertainty of $0.4 \mathrm{GeV} / c^{2}$ on $\mathrm{M}_{\mathrm{top}}$. A second, smaller contribution to this uncertainty is estimated by performing sets of pseudo-experiments in which background events are drawn not from the combined background template but from templates for one of the individual background processes, including the templates derived from QCD-enriched data. These uncertainties are estimated to be $0.3 \mathrm{GeV} / c^{2}$.

The uncertainty in the MC modeling of the b-tagging efficiency as a function of jet pT is evaluated to be $0.1 \mathrm{GeV} / c^{2}$. 
CDF Run II Preliminary (680 pb')
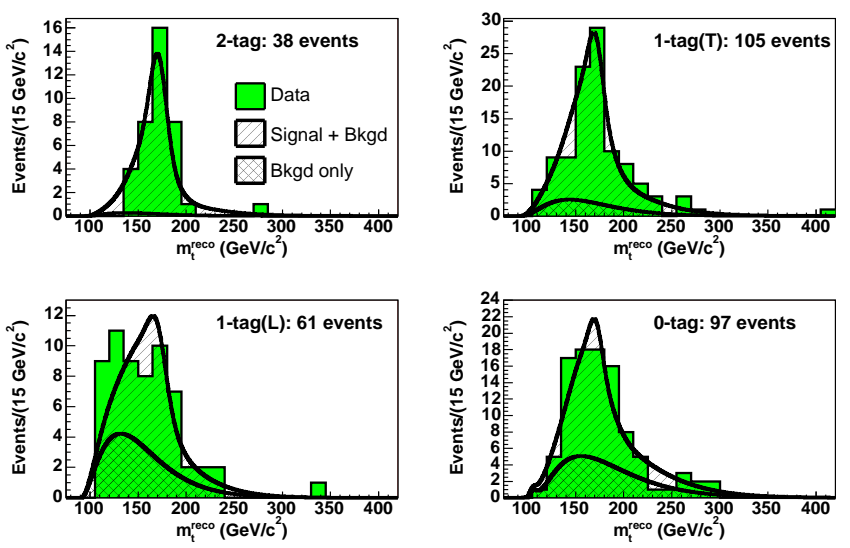

FIG. 9: $m_{t}^{\text {reco }}$ distribution in data for all subsamples. Overlayed are fitted signal and background shapes
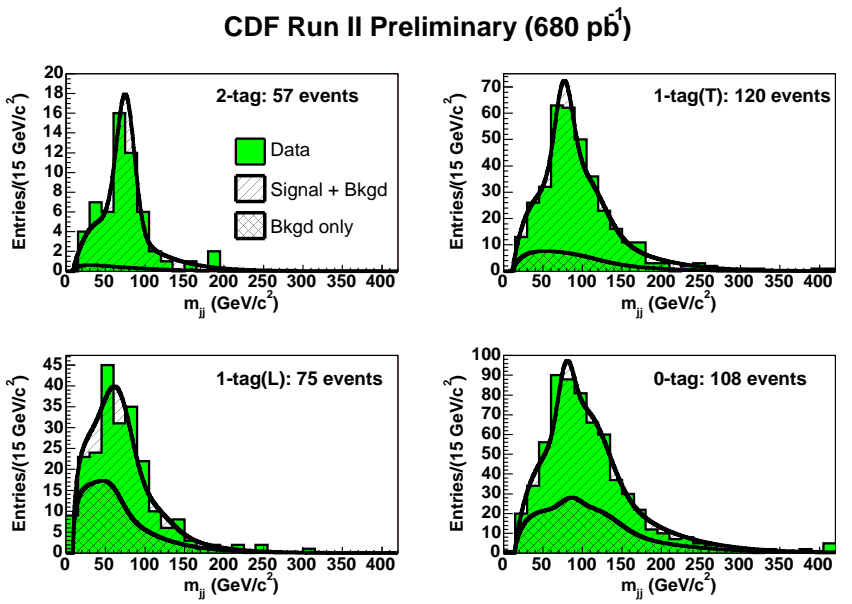

FIG. 10: $m_{\mathrm{jj}}$ distribution in data for all subsamples. Overlayed are fitted signal and background shapes

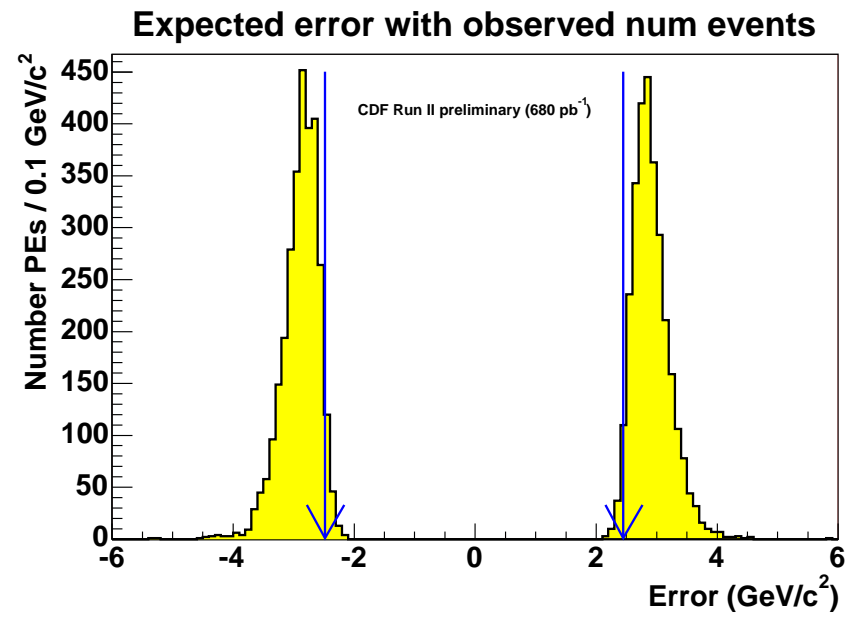

FIG. 11: Expected statistical $+\Delta_{\text {JES }}$ error on $\mathrm{M}_{\text {top }}$. Arrows highlight the value obtained in data 
Finally,the uncertainty from the limited statistics available to create the Monte Carlo templates is evaluated to be $0.3 \mathrm{GeV} / c^{2}$.

\begin{tabular}{cc}
\hline \hline Systematic Source & $\Delta M_{\text {top }}$ \\
\hline b-jet energy scale & 0.6 \\
\hline Residual JES & 0.7 \\
\hline Background JES & 0.4 \\
\hline ISR & 0.5 \\
\hline FSR & 0.2 \\
\hline Parton Distribution Functions & 0.3 \\
\hline Generators & 0.2 \\
\hline Background Shape & 0.5 \\
\hline b-tagging & 0.1 \\
\hline \hline Monte Carlo statistics & 0.3 \\
\hline TOTAL & 1.3 \\
\hline \hline
\end{tabular}

TABLE IV: summary of systematic uncertainties

\section{CONCLUSIONS}

We have measured the top quark mass to be

$$
\begin{gathered}
173.4 \pm 2.5\left(\text { stat }+\Delta_{\mathrm{JES}}\right) \pm 1.3 \mathrm{GeV} / c^{2} \\
\text { alternatively: } \\
173.4 \pm 2.8 \mathrm{GeV} / c^{2}
\end{gathered}
$$

In this measurement an in-situ Jet Energy Scale calibration employing the $W$ boson resonance has been performed. This measurement alone is more precise than the summer 2005 Tevatron average.

\section{ACKNOWLEDGEMENTS}

We thank the Fermilab staff and the technical staffs of the participating institutions for their vital contributions. This work was supported by the U.S. Department of Energy and National Science Foundation; the Italian Istituto Nazionale di Fisica Nucleare; the Ministry of Education, Culture, Sports, Science and Technology of Japan; the Natural Sciences and Engineering Research Council of Canada; the National Science Council of the Republic of China; the Swiss National Science Foundation; the A.P. Sloan Foundation; the Bundesministerium für Bildung und Forschung, Germany; the Korean Science and Engineering Foundation and the Korean Research Foundation; the Particle Physics and Astronomy Research Council and the Royal Society, UK; the Russian Foundation for Basic Research; the Comisión Interministerial de Ciencia y Tecnología, Spain; in part by the European Community's Human Potential Programme under contract HPRN-CT-2002-00292; and the Academy of Finland.

[1] A. Abulencia et al., Top Quark Mass Measurement Using the Template Method in the Lepton + Jets Channel at CDF II, Phys. Rev. D 73, 032003 (2006)

[2] The CDF collaboration., Public Conference Note "Measurement of the Top Quark Mass using the Template Method in the Lepton plus Jets Channel With In Situ $W \rightarrow j j$ Calibration at CDF-II": http://www-cdf.fnal.gov/cdfnotes/cdf7680_public_secvtx_tmtv11.pdf

[3] F. Abe et al., Phys. Rev. Lett. 74, 2626 (1995); S. Abachi et al., Phys. Rev. Lett. 74, 2632 (1995).

[4] E. Simmons, proceeding of Thinkshop on Top Quark Physics at RunII, Batavia, IL, 1998, hep-ph/9908511.

[5] G. Corcella et al., JHEP 01, 010 (2001), [hep-ph/0011263]; hep-ph/0210213.

[6] D. Acosta et al., CDF Collaboration, Phys. Rev. D 71, 052003 (2005).

[7] M.L. Mangano et al., J. High Energy Phys. JHEP 07, 001 (2003).

[8] T. Sjostrand et al., Comp. Phys. Commun. 135, 238 (2001). 